\title{
Comunicación periodística y violencias contra las mujeres. Una propuesta metodológica para su abordaje integral
}

institucional.us.es/ambitos/

\author{
Belén Zurbano-Berenguer \\ Universidad de Sevilla \\ bzurbano@us.es

\section{María del Mar García Gordillo \\ Universidad de Sevilla \\ marggordillo@us.es}

English Version: Journalistic communication and violence against women. A methodological proposal for comprehensive approach.

\begin{abstract}
Las violencias que sufren las mujeres en todo el planeta son un problema estructural aún sin erradicar y con unas cifras de incidencia alarmantes. En la lucha contra éstas una de las herramientas que se han demostrado con gran capacidad de influencia social es la comunicación. Tras décadas de investigaciones al respecto, existe un consenso académico acerca de la importancia que los mensajes de los medios de comunicación tienen en la conformación de los imaginarios colectivos y en la generación de sensibilidad social. Conocida la influencia de estos mensajes así como la necesidad acuciante de orientar los mismos hacia la erradicación de las

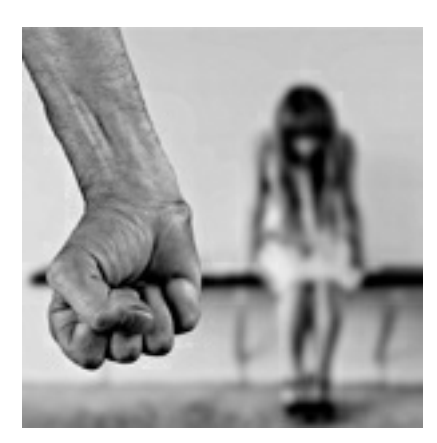

violencias, este artículo plantea una propuesta metodológica de abordaje de los

procesos mediáticos que abordan este problema. El objetivo que se persigue es, de un lado, validar una metodología integral de investigación-evaluación de los mensajes mediático-informativos y, de otro, coadyuvar a la mejora de la representación y tratamiento ético de este problema.
\end{abstract}

\section{Palabras clave}

Género, violencias, violencia de género, mujer, comunicación, periodismo, discurso.

Abstract

Violence against women worldwide are still without eradicating structural problem and alarming incidence figures. In the fight against them one of the tools that have been proven with plenty of social influence is communication. After decades of research on the subject, there is an academic consensus on the importance than the messages of the media have in shaping the collective imaginary and the generation of social sensitivity. Knowing the influence of these messages as well as the pressing need to guide them towards the eradication of violence, this paper presents a methodological proposal addressing the media processes that address this problem. The objective pursued is, on one side, validating a comprehensive methodological and evaluation research methodology of media-informational messages and, secondly, contribute to improving the representation and ethical treatment of this problem.

Keywords Gender, violence, gender violence, women, communication, journalism, discourse. 


\section{MEDIOS DE COMUNICACIÓN Y VIOLENCIAS CONTRA LAS MUJERES}

Las violencias ejercidas contra las mujeres son consideradas por la Organización Mundial de las Salud la primera causa de muerte, por encima de guerras, accidentes de tráfico o cáncer, en las mujeres de entre 14 y 44 años y ha sido declarado un "problema de proporciones epidémicas" y un "problema de salud pública" (Organización Mundial de la Salud, 2013).

Una de cada tres mujeres en el mundo son golpeadas, coaccionadas sexualmente o sufren otro tipo de abuso en su vida (UNIFEM, 2003); dos millones de niñas entre los 5 y los 15 años de edad son forzadas a prostituirse cada año y al menos 130 millones de mujeres han sufrido de mutilación genital (Observatorio de la violencia de género, 2010). Asimismo, desde el año 2009 el CEDAW viene advirtiendo que la violencia contra las mujeres a nivel mundial aumenta cada año. En Europa, los datos oficiales indican que entre el $20 \%$ y el $25 \%$ de las mujeres han sufrido violencia en alguna ocasión y que 7 mueren cada día asesinadas por sus parejas y ex parejas.

En España, esta violencia ha causado la muerte de 1047 mujeres entre 1999 y 2015 (01). Es un problema social que pervive con una fuerza inusitada y ante la que se han tomado medidas pioneras en Europa como la promulgación de una Ley Orgánica, la 1/2004 de Medidas de Protección Integral contra la Violencia de Género que protege a las mujeres en las violencias que sufren en el seno de las parejas, o la norma andaluza 13/2007, de 26 de noviembre, de medidas de prevención y protección integral contra la violencia de género, que amplía el marco de protección a violencias sucedidas tanto en el entorno afectivo, como el familiar y el social.

Ante un problema de tal envergadura y cuya erradicación es la base de una sociedad igualitaria y justa en la que las personas puedan desarrollarse en libertad y dignidad independientemente de su género es necesaria la implicación de todos los agentes sociales. Uno de los cuales los constituyen los medios de comunicación social ya que "la capacidad mediática para intervenir en la forma en que se percibe el mundo prescribiendo la posición desde la cual es adecuado observar la realidad social influye también en el funcionamiento social" (Menéndez, 2010 , p. 35). La ciudadanía basa su conocimiento sobre las violencias contra las mujeres en la información que ofrecen los medios de comunicación resultando así un gran potencial de influencia respecto a tres elementos clave: el grado de conocimiento social de este problema, el estado de la sensibilidad y la prevención de las violencias.

Es sabido desde hace tiempo que las historias influyen en la percepción pública de la realidad social, reflejan el interés público. Y juegan un papel importante en cómo las personas entienden ciertos problemas de la sociedad, especialmente la delincuencia (por ejemplo, Curran, Gurevitch y Wollacot, 1979) (...) Se ha demostrado una relación entre las representaciones del crimen y las actitudes acerca de la delincuencia, ilustrando la influencia que tienen los medios de comunicación en la opinión pública y las percepciones (Gilliam, lyengar, 2000, 2005; Holbert, Shah y Kwak, 2004) (Wozniak, J. y McCloskey, K., 2010, p.938).

La importancia es tal que en la Ley Orgánica 1/2004 se establece que uno de los ámbitos en los que debe incidirse para la lucha contra esta lacra es el de los medios de comunicación (capítulo II, artículos 13 y 14). Así, se plantea en la Ley la necesidad de que exista un contexto general de igualdad y respeto en las representaciones de los géneros y uno particularmente sensible y cuidado en las noticias sobre esta violencia. Lo que ha dado lugar en los últimos años a un gran desarrollo académico y deontológico sobre el tratamiento de la violencia de género en los medios de comunicación social.

En esta materia, la Comunidad Andaluza y en concreto el Instituto Andaluz de la Mujer (IAM) ha mantenido siempre una actitud pionera, proactiva y valiente en la defensa de la dignidad de los contenidos mediáticos sobre esta violencia. Ya en 1999 elaboró el primero de los documentos orientativos sobre cómo debían los medios de comunicación abordar el tratamiento de una cuestión tan delicada y que aún sigue siendo un referente para los diversos manuales sobre cómo realizar una correcta labor periodística en esta materia. Asimismo, el Ayuntamiento de Sevilla consciente de la labor fundamental de los medios de comunicación social ha realizado numerosos esfuerzos para mejorar la visibilidad de la violencia en los medios y la representación igualitaria y respetuosa de la mujer en la comunicación.

En la actualidad, existen más de una decena de decálogos, recomendaciones y manuales de buenas prácticas 
que proliferan cada año intentando paliar el maltrato de estas informaciones por parte de los media a los que se acusa de tratamientos defectuosos y poco responsables. Sólo entre noviembre de 2010 y enero de 2011 y en nuestro contexto más inmediato vieron la luz dos documentos guía para abordar informativamente la violencia de género: el Acuerdo Marco del compromiso de los medios de comunicación y periodistas de Extremadura contra la violencia hacia las mujeres (Asociación de la prensa de Mérida, Asociación de la prensa de Cáceres, Asociación de la prensa de Badajoz, 2010) y la obra Cómo informar sobre violencia contra la mujer en las relaciones de pareja (Noblejas y Arribas, 2011). Y éstos son solo los más recientes ya que existen una gran variedad de decálogos, recomendaciones y demás suerte de documentos que coinciden en los valores y prácticas a seguir.

Por ello, una mirada retrospectiva sobre qué esfuerzos se han realizado en materia de violencia, género y medios de comunicación es fundamental para conocer el estado de la cuestión sobre la deontología periodística y sentar las bases de la actualización de los principios que ya inspiraron al IAM en 1999. Por ello, una revisión de las propuestas existentes parece oportuna en un contexto en el que deben continuar los esfuerzos por mejorar la comunicación pública de este drama social ya que son muchas las mejoras que aún cabe implementar en el tratamiento noticioso de esta violencia: la práctica habitual de descripción reiterada del modus operandi, la ausencia de fuentes expertas o de testimonios de testigos, familiares y entorno, desaconsejados por todos los manuales deontológicos salvo excepciones, las representaciones parciales de las víctimas como sujetos pasivos y sin agencia (Bengoechea, en el año 2000 ya denuncia la incapacidad mediática de concebir simultáneamente la violencia masculina y la agencia femenina).

(...) La forma en que se informe de la VAWG (02) es a menudo inexacta, minimiza las acciones de los agresores, y sitúa la culpa en la víctima u otras personas reforzando los mitos y estereotipos. De esta manera, las representaciones son perjudiciales, contribuyendo y reforzando el contexto cultural en el que la VAWG ocurre (AAVV, 2012, pág.7).

El discurso mediático interviene en la forma de percibir el mundo, prescribiendo la posición desde la cual es adecuado observar la realidad social (Menéndez, I., 2010, p.35)

En general, puede hablarse de la influencia de los medios de comunicación en materia de violencia contra las mujeres en tanto que:

\section{Capacidad de agenda.}

Tienen la posibilidad de informar sobre los casos o no hacerlo, tal y como ocurría hasta la década de 1990 en España, donde la información no aparecía o bien aparecían los casos más sangrantes (como el denominado "crimen de las niñas de Alcàsser").

\section{Capacidad sobre los enfoques.}

Los medios de comunicación deciden el enfoque o marco de las noticias. El que ha sido más habitual hasta el momento es el encuadre como suceso y el propuesto desde la deontología del tratamiento como problema social y atentado contra los Derechos Humanos. De los medios depende la especialización de la información, la calidad de las fuentes o los espacios de inclusión de las noticias sobre violencia de género.

\section{Capacidad sobre la conceptualización.}

Es la capacidad definitoria de la violencia de género por parte de los medios. Supone el reconocimiento de la heterogeneidad de manifestaciones y escenarios de las violencias por razón de género o, por el contrario, su reducción conceptual.

\section{Capacidad de influencia sobre la terminología.}

En la nominalización del problema existen matices no poco importantes ya que suponen un marco interpretativo del fenómeno. La forma de nombrar las realidades influye además en la conformación de éstas por lo que las herramientas lingüístico-conceptuales para referirse a las agresiones de género no carecen de importancia en 
el discurso público. Las terminologías llevan aparejadas ideologías y conceptualizaciones ulteriores como, por ejemplo, que la "violencia de género" es definida en España por la relación afectiva entre víctima y victimario aislando otras agresiones de naturaleza patriarcal que quedan sin denominación alguna.

\section{Capacidad sobre los efectos.}

Los mensajes mediáticos sobre violencia han sido objeto de diversos estudios y se han generado diversas teorías explicativas con respecto a sus efectos (03). La sensibilidad ciudadana para con las violencias que sufren las mujeres a diario es clave fundamental para la erradicación progresiva de esta grave lacra social. Es a través de los imaginarios y mentalidades que se construye el rechazo colectivo a este problema anclado en el androcentrismo y el patriarcado. Los medios de comunicación, como mediadores sociales y conformadores de opinión pública poseen en este ámbito una importancia capital ya que no sólo visibilizan las violencias y las condenan sino que pueden influir en la detección, la prevención y el rechazo/denuncia de las agresiones machistas.

En este sentido y por todo lo expuesto se considera fundamental, de un lado, actualizar los consensos deontológicos ya existentes, y, por otro, concretar un protocolo marco de investigación integral que evalúe los discursos mediáticos sobre las violencias de género de forma integral.

\section{UNA MIRADA INTEGRAL A LOS DISCURSOS MEDIÁTICOS}

La metodología de investigación que aquí se propone pretende, por un lado, superar los límites del análisis unifocal del análisis de contenido (tan recurrente y por supuesto oportuno, aunque no suficiente, en materia de análisis periodístico) favoreciendo una mirada integral al proceso de la comunicación periodística. Y, por otro, enriquecer las posibilidades de comprensión del proceso periodístico-informativo en sí mismo para favorecer la implementación de cambios y mejoras en la representación y tratamiento de las violencias que sufren las mujeres.

Que el periodismo debe ser un aliado en la lucha por la erradicación de las violencias es la premisa de partida de esta propuesta metodológica que, sin embargo, parte del compromiso social de las autoras, convencidas de que la Academia debe favorecer análisis complejos que además de denunciar las desviaciones existentes propongan modelos y pautas de mejora realistas y aplicables que partan de un conocimiento profundo y completo del sistema de producción discursiva. Es desde este empeño desde el que las autoras ofrecen su propuesta de metodología de abordaje integral para su discusión y debate público con el objetivo de hallar un método que no sólo evidencie o radiografíe la realidad de los discursos mediáticos sino que los comprenda para poder mejorarlos.

La propuesta de estudio planteada para la matriz medios- violencias contra las mujeres pretendería responder, de forma general, al "análisis delas características y cambios en el discurso informativo-noticioso sobre las violencias contra las mujeres y su valoración por parte de las instituciones emisoras y las víctimas de las violencias narradas". Es decir, el método de estudio favorece la identificación de las características básicas de los mensajes periodísticos para, a partir de ellos, analizar las condiciones de elaboración de dichos mensajes (la participación e influencia del periodista y del medio ambos como partes de la institución emisora) y la recepción-interpretación por los ciudadanos/as cuidando, especialmente, la voz de la mujeres supervivientes de violencias.

Este enfoque se fundamenta en la necesidad de conocer, para comprender y para cambiar, el discurso periodístico de las VVCMM desde diferentes planos: el de la información publicada, el de las instituciones productoras de los mensajes y el de las mujeres violentadas que reciben dichos mensajes y la ciudadanía como receptora en su conjunto. En este sentido, puede decirse que el objeto material de la investigación lo constituyen los medios de información, y, en concreto, de una parte de ellos (textos, emisiones o cualquiera de los formatos existentes) convertidos en unidades objeto de análisis.

La investigación, por tanto, se organizaría y definiría en tres planos de análisis: el del mensaje informativo, el de 
la producción noticiosa y el de la recepción. De este modo, los objetivos de cada uno de los niveles analíticos así como las unidades de observación se diferenciarán atendiendo a la especificidad y profundidad del análisis perseguido.

Este planteamiento metodológico está influido por las orientaciones aportadas por Wallace (1976) en su proceso científico de la investigación social. Del conocido como el "Círculo de Wallace" se toman las concepciones críticas de la investigación (en tanto que no es finita, no está acabada y debe someterse a una revisión continua), es empírica (pues toma sus resultados de la experiencia) y tiene un carácter circular (la interacción continuada entre la teoría y las experiencias empíricas es la que permite la generación del conocimiento). Desde un enfoque metodológico cualitativista el modelo de investigación integral/ circular propuesto en esta comunicación es deudor del los presupuestos constructivistas de investigación. El diseño de la investigación en planos comunicativos y el diálogo pretendido entre ellos no es más que el efecto de la construcción simultánea del análisis y la recogida de los datos pues, exceptuando el vaciado de prensa (en el que ya se iba construyendo paralelamente el "texto interpretativo provisional" descrito por Ruiz, J.I., 1996, p. 215) en el resto de las unidades no existen espacios delimitados entre la recolección de datos y sus análisis.

Del mismo modo, se encuentran en la aplicación de las técnicas de investigación cualitativa y en los fundamentos generales del diseño de la investigación preceptos de la Teoría Fundamentada (Strausser, A. y Claser, B, 1970) en tanto que se persigue la generación de una teoría a partir de la sistematización y codificación de los datos mediante una comparación constante para el establecimiento de las relaciones entre los datos y los conceptos teóricos.

La investigación que se plantea, de corte empírico, persigue, a partir de marcos teóricos sólidos, profundizar en la teoría, renovarla y completarla. Una teoría que contribuya a generar los cambios efectivos en la erradicación de las violencias patriarcales. Otros puntos de encuentro con los presupuestos de esta Teoría es la utilización del llamado "muestreo teórico" y el método de trabajo propuesto por Strauss (1970) basado en la recogida de datos, codificación y reflexión analítica en notas.

Este planteamiento, a pesar de trabajar a partir de metodologías tradicionalmente masculinizadas insiste en autodefinirse metodológicamente feminista ya que su objetivo es apropiarse de las herramientas, transformarlas adaptándolas a las nuevas necesidades y utilizarlas para la eliminación de las opresiones de género.

Los análisis feministas se centran en desarrollar teorías y modelos de intervención que reflejen adecuadamente las experiencias de las mujeres (...) este cambio metodológico se concibe, además, como una cuestión ética y política en cuanto que la epistemología propuesta pretende servir a fines prácticos y contribuir a la solución del problema (Zurilla, M.A. y Domínguez, P., 2011, p.27).

Las críticas a las metodologías tradicionales han partido de su sobresujeción de su situación absoluta dentro de la cosmovisión del poder y frente a ellas las aportaciones feministas han intentado corregir las desviaciones que permitan ampliar los objetos de estudio y complejizar las herramientas y los análisis adaptándolas a las realidades antes no contempladas (04).

Desde una perspectiva de género, los investigadores han observado que las metodologías, epistemologías y métodos "tradicionales" no son científicamente "objetivos" sino todo lo contrario: por lo general ignoran el conocimiento de las mujeres mostrando un sesgo hacia la perspectiva masculina. En respuesta a esto, las metodologías utilizadas para la investigación sobre la mujer en el desarrollo se han realizado a partir de las críticas hacia un sexo y clase determinados y los prejuicios raciales encontrados en las metodologías de investigación "tradicionales", así como en las perspectivas de desarrollo dominantes (Beetham, G. y Demetriade, J. , 2007, p. 199).

El estudio del mensaje informativo se ha considerado fundamental por constituir el objeto comunicativo materializado, tangible, a partir del cual evidenciar y comprender los usos, tratamientos y representaciones sociales que se hacen de las VVCMM.

\subsection{El diseño metodológico}


Para elaborar el diseño metodológico de la investigación hay que partir, por tanto, del eje inicial de la representación mediática (el tradicional análisis del contenido o discursos informativos) para luego realizar las evaluaciones en las instituciones emisoras y receptoras. La siguiente Figura 1 plantea un esquema sintetizado del proceso seguido en la investigación de referencia (Zurbano, B., 2015) en la que se puso en práctica esta metodología de investigación integral:

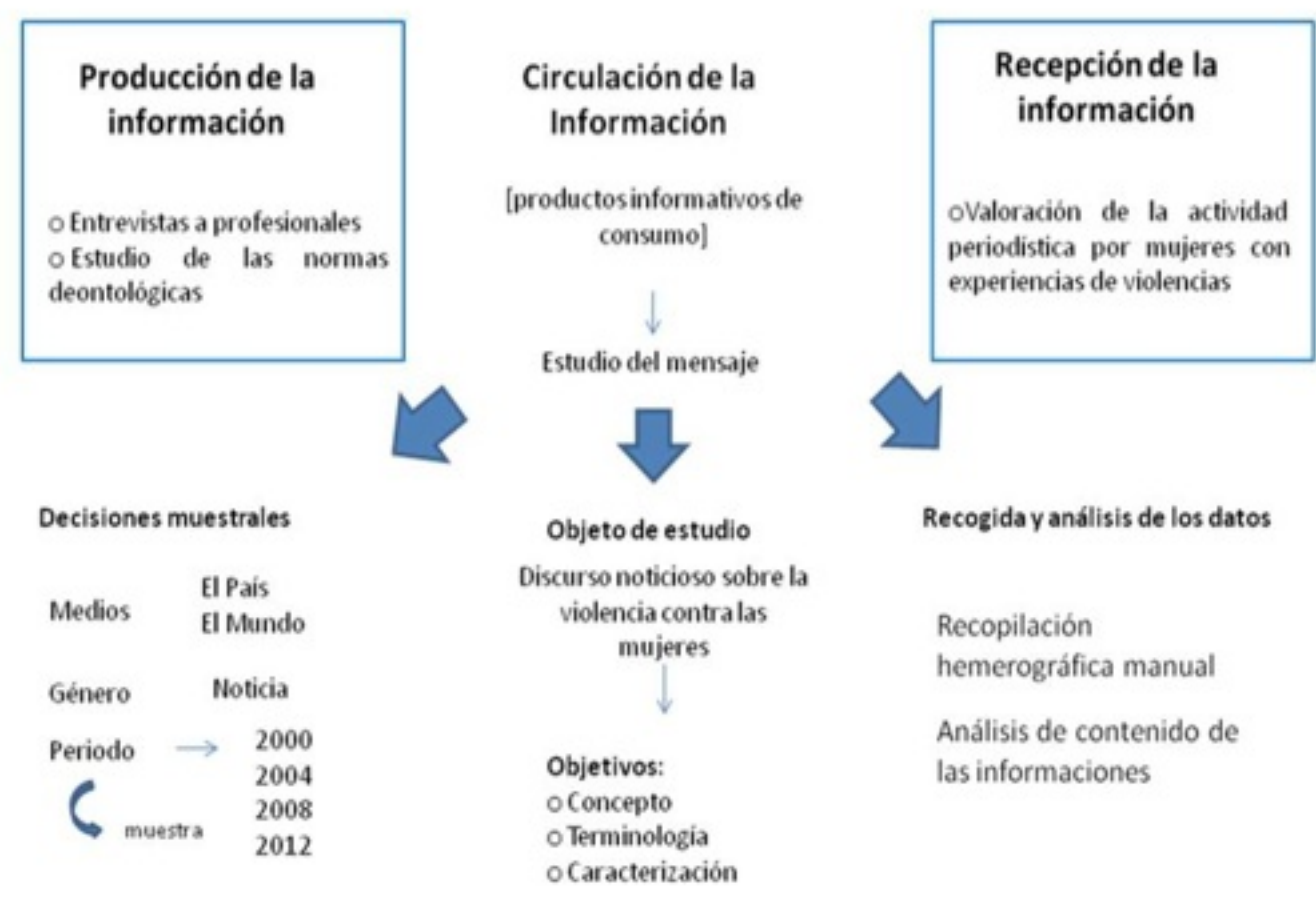

Estudio sincrónico - Comparativa entre diarios- Identificación de cambios L.0.2004

La propuesta metodológica plantea que deben existir, cuanto menos, 3 unidades de análisis claramente diferenciadas y con potencialidades interpretativas acumulativas. A saber: el texto noticioso (entendido el "texto" de manera amplia (05)), el discurso de los profesionales y la valoración de la ciudadanía- mujeres violentadas. Como mínimo, para comprender el sentido e interpretación así como su oportunidad y adecuación de los discursos periodísticos sobre VVCMM, estos tres elementos deben estar presentes. Y podrían ampliarse. Poner en relación la noticia con sus productores y con las personas receptoras es la base mínima para una metodología integral de abordaje pero la riqueza de los análisis puede multiplicarse si se atendiese también a cuestiones macro, como la autoimagen profesional, el estado de la sensibilidad social, el contexto económicosocial de los productores de información desde su rol de asalariados o elaborando diferentes estrategias de investigación en las instituciones receptoras a partir de una estratificación por nivel de conocimiento y/o cercanía al problema, por ejemplo.

Además, cada uno de los planos de análisis debe posee sus propios objetivos específicos diseñado a partir de la revisión de la literatura existente y de los resultados exploratorios del plano anterior dando sentido pragmático a la "circularidad" semántica de la metodología de inspiración. En la investigación de referencia (Zurbano, B., 2015) las unidades de análisis del trabajo de investigación fueron las que siguen:

Figura 2. Resumen de los objetivos y las unidades de análisis asociados. 


\begin{tabular}{|c|c|c|}
\hline Plano comunicativo & Objetivos & Unidades de análisis \\
\hline \multirow{5}{*}{ Circulación (producto discursivo) } & $\begin{array}{l}\text { 1) Reconocer, cuantificar y analizar las formas de } \\
\text { abordaje terminológico }\end{array}$ & \multirow{5}{*}{ Noticias (1) } \\
\hline & $\begin{array}{l}\text { 2) Estudiar los principales rasgos de las noticias } \\
\text { de VVCMM }\end{array}$ & \\
\hline & $\begin{array}{l}\text { 3) Comparar la representación de las WVMM con } \\
\text { las definiciones teóricas y legislativas al uso }\end{array}$ & \\
\hline & $\begin{array}{l}\text { 4) Observar la distribución temática atendiendo a } \\
\text { la multiplicidad de formas de violencia }\end{array}$ & \\
\hline & $\begin{array}{l}\text { 5) Identificar posibles cambios en usos } \\
\text { terminológicos y en caracterización y } \\
\text { conceptualización. }\end{array}$ & \\
\hline \multirow{4}{*}{ Emisión } & $\begin{array}{l}\text { 6) Identificar e inventariar los códigos } \\
\text { autorregulatorios. }\end{array}$ & \\
\hline & $\begin{array}{l}\text { 7) Estudiar los presupuestos ético-deontológicos } \\
\text { a fin de establecer los presupuestos éticos } \\
\text { comunes }\end{array}$ & $\begin{array}{l}\text { Códigos normativo- } \\
\text { deontológicos (2) }\end{array}$ \\
\hline & $\begin{array}{l}\text { 8) Profundizar en el grado de formación de los } \\
\text { profesionales y su nivel de concienciación y } \\
\text { sensibilidad }\end{array}$ & \multirow[t]{2}{*}{$\begin{array}{l}\text { Profesionales de los } \\
\text { medios de comunicación } \\
\text { (3) }\end{array}$} \\
\hline & $\begin{array}{l}\text { 9) Conocer la valoración de los periodistas sobre } \\
\text { la labor mediática y sus rutinas productivas }\end{array}$ & \\
\hline \multirow[t]{2}{*}{ Recepción } & $\begin{array}{l}\text { 10) Reflexionar sobre la valoración de la actividad } \\
\text { periodistica en la materia y la percepción del } \\
\text { cambio sufrido en el tratamiento por los usuarios } \\
\text { de la comunicación }\end{array}$ & \multirow[t]{2}{*}{$\begin{array}{l}\text { Mujeres victimas de } \\
\text { WVCMM (4) }\end{array}$} \\
\hline & $\begin{array}{l}\text { 11) Valorar las recomendaciones éticas existentes } \\
\text { para tratar la VVCMM en los medios de } \\
\text { comunicación }\end{array}$ & \\
\hline
\end{tabular}

Fuente: elaboración propia.

\subsection{Las técnicas de investigación}

Para realizar una investigación fundamentada en las tres unidades básicas expuestas (textos noticiosos, instituciones emisoras e instituciones receptoras) se plantea la combinación de técnicas tradicionalmente cuantitativas (06) con otras de corte cualitativo.

Para el análisis de los textos noticiosos se emplearía el Análisis de Contenido (AC) que además ya ha sido aplicada previamente en estudios relacionados con la violencia y su predicción, como por ejemplo en Gerbner (1995) y Signorielli y Morgan (1996) (Iguartúa, J.J. y Humanes, M.L., 2010) como también en Caro (2011). Y para poner estos en relación con las instituciones emisoras (para responder a porqué son estos los resultados 
discursivos de la operación interpretativa del periodismo) y con la recepción (¿qué interpretación realizan los y las usuarios de medios?, ¿qué valoración realizan de los mensajes?, ¿qué efectos tienen? (07)) se atendería a la entrevista semiestructurada con los periodistas y al grupo de discusión con las mujeres violentadas así como con otros grupos de interés.

La utilización del AC para el estudio de la producción noticiosa responde a los campos tradicionales de aplicación de esta técnica en tanto que: en primer lugar, describe la realidad mediada de las VVCMM; en una segunda instancia, sirve para comprobar la relación entre la representación del discurso periodístico y el discurso jurídico, para, en un tercer estadio, evaluar "la coherencia entre la representación ofrecida en los medios y la situación existente en la realidad” (Iguartúa, J.J. y Humanes, M.L., 2010, p. 79).

Aunque no es necesario por lo conocido de esta técnica adentrarse en su evolución histórica (consultar para ello Piñuel, J.L, 2002; Krippendorff, K., 1990; Bardin, L., 1986) sí que se proponen algunas de las definiciones básicas que se manejan y que se suscriben en este estudio. Por ejemplo, la de Bardin, que define el análisis de contenido como el (...) conjunto de técnicas de análisis de comunicaciones tendente a obtener unos indicadores (cuantitativos o no) por procedimientos sistemáticos y objetivos de descripción del contenido de los mensajes, permitiendo la inferencia de conocimientos relativos a las condiciones de producción/ recepción (variables inferidas) de estos mensajes (1986, p.32).

O la de Krippendorff: Técnica de investigación destinada a formular, a partir de ciertos datos, inferencias reproducibles y válidas que puedan aplicarse a su contexto (1990, p.29).

De la primera, se ha tomado la recomendación de la descripción únicamente como primera fase para, con los resultados del análisis de contenido, inferir determinados problemas o ausencias a partir de los cuales adentrase en las percepciones desde las instituciones de producción de los mensajes y en las valoraciones desde la recepción (en las que se ahondarán en un primer caso con los periodistas mediante entrevistas, y, en un segundo caso, mediante la técnica del grupo de discusión con grupos de receptores estratégicos).

De la proposición de Krippendorff se ha asumido la necesidad de que, si bien los resultados obtenidos de una muestra intencional no pueden ser al cien por cien representativos, sí pueden ser aplicables a su contexto comunicativo como referentes de una normalidad periodística para la que el análisis de una pequeña muestra pueda servir de ejemplo de un continuum.

La entrevista (cuya tipología específica variará dependiendo de los objetivos y características de las investigaciones marco) se plantea con una doble finalidad: la obtención de información (cómo no) y la legitimación de los profesionales como actores sociales de relevancia en los estudios sobre discursos periodísticos. Esta propuesta asume los periodistas deben gozar, pues son tanto merecedores de ello como agentes potenciales de cambio social, de un espacio donde darles voz en las revisiones teóricas sobre el fruto de su trabajo. Todo ello desde una perspectiva académica dialógica para con los objetos/los sujetos de estudio y consecuentemente, con los productores-autores de dicho objeto socio-cultural como son los procesos y discursos periodísticos.

Así, la entrevista como técnica de investigación frente a otros usos, por ejemplo clínicos, se ha entendido como el contrato comunicativo en el que, a pesar de los elementos externos intrínsecos a los roles diferenciados de entrevistadora y entrevistados, existe un nexo común de conocimiento e interés mutuo: el trabajo profesional periodístico. Frente a otro tipo de técnicas, la entrevista se ha considerado la idónea como método de trabajo, ya que persigue recoger las propias valoraciones, explicaciones causales e interpretaciones intersubjetivas del fenómeno discursivo noticioso por los profesionales implicados.

En particular, como decíamos, se ha empleado y se propone la entrevista cualitativa enfocada o focalizada (Merton, R.K., 1946, 1987) estandarizada no programada (o semiestructurada para Corbetta, P., 2007) ya que persigue la obtención de determinados datos (se focaliza en determinados temas), sigue una hoja de ruta (se parte de un conjunto de preguntas o cuestiones a tratar con el entrevistado) y formula las cuestiones adaptadas al contexto, persona y discurso que se mantiene y no existe un orden de inserción de los elementos en la conversación (no está programada). Además, esta forma de entrevista pertenece a los que Lewis denomina "entrevista especializada y a élites" (en Valles, M.S., 2002) entendiendo, como el autor, que las élites, lejos de 
ser personalidades relevantes en el mundo social son "expertos o sujetos bien informados". De este modo, los periodistas con quienes se trabaja operarían no solamente como sujetos bien informados y conocedores del mundo periodístico por su especialización profesional sino además como expertos en tanto que productores de las noticias sobre el que se les ha entrevistado.

Por último, una vez establecido qué plantea el discurso noticioso y el porqué de éste desde la explicación de sus promotores, es fundamental conocer la valoración por parte de los usuarios y usuarias, o, incluso, los efectos sobre éstos y éstas. Es importante resaltar que en este punto lo más interesante es, de un lado, conocer los discursos grupales y la afectación colectiva más que la interpretación personal de un sujeto frente a un texto; y, de otro, reseñar la potencialidad de establecer diferentes analíticas (y por tanto procedimentales) entre usuarios-usuarias y mujeres violentadas o con experiencias de violencia. Por ello, la técnica que se propone para cerrar este análisis integral es la del grupo de discusión: "un método cualitativo con el objetivo principal de describir y comprender las percepciones, interpretaciones y creencias de una población seleccionada para alcanzar la comprensión de un tema en particular desde la perspectiva de los participantes de los grupos" (Liamputtong y Ezzy, 2005). Su carácter cualitativo y su focalización en valoraciones y percepciones son los motivos por los que se torna en una técnica muy apropiada para trabajar sobre los objetivos de valoración de la actividad periodística, percepción de una posible evolución e interpretación de las recomendaciones éticas. Si bien, hay que reconocer, esta técnica se ve superada para un análisis de efectos. Para alcanzar este objetivo loable y aún poco explorado (cómo afectan a las propias mujeres violentadas los discursos sobre las violencias por razón de género) habría que realizar un diseño ad-hoc más complejo y específico.

A pesar del uso como sinónimo que suele dársele en Ciencias Sociales al grupo de discusión y al focus group, hay que destacar que el grupo de discusión presenta una mayor flexibilidad y apertura así como un estilo de conducción menos directiva que el focus (Gutiérrez, 2008). Además, el uso del grupo de discusión ha sido generalizado en la investigación académica para dar voz a grupos marginalizados o al margen del poder, por lo que se ha entendido útil para trabajar con identidades estigmatizadas como son las de las mujeres con una experiencia de violencia de este tipo.

La necesidad de obtener datos cualitativos (interpretaciones, valoraciones, estado de las percepciones) de un grupo estigmatizado hace de esta técnica la herramienta de obtención de información más apropiada teniendo en cuenta que algunas de las potencialidades de esta herramienta son:

1. Obtener conocimiento profundo o sobre temas sensibles.

2. Facilitar la discusión de temas incómodos o difíciles de tratar en encuentros individuales.

3. Ayudar a expresar las opiniones y experiencias propias a partir de la escucha de las ajenas.

4. Hacer visible el campo semántico en que se encuadra este grupo. Se ha buscado específicamente su discurso como representantes de un colectivo. A través de sus voces se ha perseguido visibilizar los matices del campo semántico que les es propio a este grupo de mujeres y que representan a las mujeres que viven y ocupan posiciones estructurales y vitales similares en la sociedad (08).

\subsection{Una llamada a la multidisciplinariedad de los equipos de investigación}

La propuesta metodológica que se ha realizado aquí se caracteriza por el uso de diversas técnicas de investigación para las que los comunicólogos y comunicólogas no contamos siempre en un nivel de cualificación suficiente. El estudio de los procesos de comunicación (con sus diferentes niveles y enfoques posibles), aunque siempre complejos y favorables a un abordaje multidisciplinar, en ocasiones queda focalizado por las técnicas de estudio tradicionalmente empleadas para el estudio de los textos y discursos: el análisis de contenido y el análisis del discurso (y sus variantes terminológicas y epistemológicas).

En particular, el estudio de los discursos sobre violencias y medios y sobre todo en España ha estado dominado por un estudio de las representaciones basado en los contenidos y la ideología y apoyado en las técnicas anteriormente mencionadas. Habiendo realizado previamente un alegato a la importancia de los textos y discursos en esta propuesta de investigación integral y por tanto e indirectamente reconociendo la posición fundamental de sus técnicas asociadas es menester igualmente hacer un llamamiento a la interdisciplinariedad 
del campo de estudio (la comunicación, las VVCMM). Llamamiento que quiere hacer valer la importancia de las técnicas de investigación en comunicación y de los profesionales de la misma, pero también la necesidad de compartir con otras disciplinas (y por tanto con sus expertos y expertas en otras técnicas de investigación) abordajes alternativos y complementarios que ayuden a conformar una comprensión global de los procesos comunicativos que vayan más allá de sus resultados evidentes: los textos. Un abordaje integral y multidisciplinar es necesario para plantear claves de comprensión sobre la totalidad del proceso de comunicación que evidencie la relación entre las condiciones de conocimiento y sensibilidad que inciden la producción final de textos como los que encontramos cada día en la prensa hasta la importancia significativa que conceden mujeres supervivientes de violencias a éstos. Todo ello para favorecer planteamientos alternativos que favorezcan una comunicación responsable y estratégica que coadyuve a la erradicación de las violencias que no serán posibles sin la necesaria multidisciplinariedad de técnicas y expertos/as.

\section{DISCUSIÓN Y CONCLUSIONES}

La propuesta metodológica que se ha planteado, basada en la necesidad de un abordaje multidimensional de la comunicación periodística de las VVCMM que integren en los estudios académicos a las instituciones emisoras y receptoras de forma sistemática, ya ha sido aplicada de forma exploratoria en una tesis doctoral (09). $Y$ ha permitido comprobar la riqueza de las interrelaciones que se operan entre los diferentes planos de análisis.

Esta primera aproximación ha permitido evidenciar que es posible obtener resultados contextualizados e interrelacionados simultáneos en los tres planos clásicos de la comunicación de masas (emisión, circulación y recepción). Y que la potencialidad dialógica de trabajar con los hallazgos de los diferentes planos y sus unidades/sujetos de estudio sincrónicamente permite enriquecer los análisis e interpretaciones sobre los discursos mediáticos.

Además de su valor de contenido, la metodología integral de la investigación tiene una carga ideológica intrínseca, la de reconocer y valorizar a las instituciones emisoras de mensajes periodísticos y a los propios profesionales, así como a los y las usuarios/as de medios de comunicación, y fundamentalmente a las mujeres supervivientes de violencias, como agentes clave para el desarrollo de teorías explicativas ya sea en cuanto a trabajos sobre políticas de representación o en cualquier otro orden de las teorías de medios.

Un análisis parcial basado únicamente en uno de los planos comunicativos va a favorecer siempre resultados sesgados y descontextualizados de un proceso que, por definición, es siempre circular y compete no sólo al producto acabado o texto emitido/publicado, al modo de elaboración o a las casuísticas de su recepción, sino a todos y cada uno de estos elementos en su compleja relación.

\section{REFERENCIAS BIBLIOGRÁFICAS}

AAVV (2012). Just the Women. An evaluation of eleven British national newspapers' portrayal of women over a two week period in September 2012, including recommendations on press regulation reform in order to reduce harm to, and discrimination against women. Recuperado el 31 de febrero de 2014 en http://goo.gl/NcZTxm

Bardin, L. (1986). Análisis de contenido. Madrid, España: Akal.

Beetham, G. y Demetriade, J. (2007). Feminist Research Methodologies and Development: Overview and Practical Application. Gender and Development, 15(2), 199-216.

Bengoechea, M. (2000). En el umbral de un nuevo discurso periodístico sobre violencia y agencia femenina: De la crónica de sucesos a la reseño literaria. Cuadernos de Información y Comunicación, 5, 9-22.

Corbetta, P. (2007). Metodología y técnicas de investigación social. Madrid, España: McGraw- Hill.

Caro, F.(2011). La satisfacción de las necesidades informativas de las mujeres. Análisis de la empresa 
periodística desde la perspectiva de género (Memoria de investigación INVM PR04107). Recuperado de http://bit.ly/1BiNPli

Fernández, A. y Noblejas, M. (2011). Cómo informar sobre violencia contra la mujer en las relaciones de pareja. Madrid, España: Escuela de Periodismo y Comunicación.

Gutiérrez, J. (2008). Dinámica del grupo de discusión. Cuadernos metodológicos. Madrid, España: Centro de Investigaciones Sociológicas (CIS).

Igartua, J. J. y Humanes, M. L. (2010). Teoría e investigación en comunicación social. Madrid, España: Síntesis. Krippendorff, K. (1990). Metodología de análisis de contenido. Teoría y práctica. Barcelona, España: Paidós.

Menéndez, M. I. (2010). Representación mediática de la violencia de género. Análisis de la prensa balear 20042008. Palma de Mallorca, España: Edicions Universitat de les Illes Balears.

Merton, R. K. y Kendall, P. L. (1946). The focused interview. American Journal of Sociology 51(6), 541-557.

Observatorio de la Violencia de Género (2010). Aumenta en el mundo la violencia de las mujeres según la ONU. Observatorio de la Violencia de Género. Recuperado el 9 de octubre de 2014 en http://goo.gl/5DkAOI

Organización Mundial de la Salud (2013). Global and regional estimates of violence against women: prevalence and health effects of intimate partner violence and non-partner sexual violence. Suiza:Organización Mundial de la Salud.

Osborne, R. (2009). Apuntes sobre violencia de género. Barcelona, España: Ediciones Bellaterra.

Piñuel Raigada, J. L. (2002). Epistemología, metodología y técnicas del análisis de contenido. Estudios de Sociolingüística, 3(1), 1-42.

United Nations Development Fund for Women (UNIFEM) (2003). Not a minute more. Ending violence against women. Nueva York, EEUU: United Nations Development Fund for Women.

Valles, M. S. (2002). Técnicas cualitativas de investigación social. Reflexión metodológica y práctica profesional. Madrid, España: Síntesis.

Van Dijk, T. (1990). La noticia como discurso. Comprensión, estructura y producción de la información. Barcelona, España: Paidós.

Van Dijk, T. (2003 Liamputtong, P. y Ezzy, D. (1995). Qualitative research methods. Australia: Oxford.

Wozniak, J. y McCloskey, K. (2010). Fact or fiction? Gender issues related to newspaper reports of intimate partner homicide. Violence Against Women, 16(8), 934-952.

Zurbano, B. (2015). Discurso periodístico y violencias contra las mujeres. Aproximaciones a la construcción y valoración de los mensajes informativos en la prensa de referencia en España (2000-2012). Trabajo inédito.

Zurilla, M. A. y Domínguez, P. (2011). Violencias contra las mujeres, un enfoque jurídico . Oviedo, España: Septem Ediciones

(01) Fuente: Delegación del Gobierno para la Violencia de Género. Ministerio de Sanidad, Servicios Sociales e Igualdad.

(02) Violence against women en la nomenclatura internacional. 
(03) Un buen resumen de éstas teorías y sus autores de referencia puede encontrarse en Igartúa y Humanes (2010) quienes realizan la siguiente clasificación de constructos explicativos sobre los efectos de los contenidos mediáticos sobre violencia y pornografía: la hipótesis de la catarsis, la teoría de la imitación o modelado simbólico, la desinhibición y legitimación de la violencia, la preactivación o efecto primming, el modelo de la desensibilización, el de la insensibilidad sexual, la teoría de la transferencia de la excitación y el reciente "análisis contextual".

(04) Hay que hacer notar que no sólo desde la perspectiva feminista se ha cuestionado la epistemología tradicional.

(05) Ver Van Dijk, 2003.

(06) Si bien no es la intención de este trabajo adentrarse en la disyuntiva cualitativo-cuantitativo tradicional en el debate sobre el análisis de contenido, sí ha parecido oportuno reseñar esta perspectiva poco usual, y que aquí se comparte, de que el análisis de contenido no es una técnica únicamente cuantitativa (también Krippendorff, L., 1990 y Porta, L. y Silva, M., s.f.). No sólo el diseño de las unidades de análisis y la selección de la muestra sino, sobre todo, la elaboración de las variables de estudio son procesos eminentemente cualitativos. Además, el enfoque de contingencias que se utiliza para el análisis es una operación fundamentalmente de corte interpretativo que únicamente sustenta sobre datos puros el cruce matemático de las magnitudes de estudio. De igual modo, el AC con que se ha trabajado la producción deontológica es de carácter cualitativo siguiendo el proceso "del campo al texto y de éste al lector" (Ruiz, J.I., 1996, p. 201). Otras definiciones como la de Piñuel Raigada inciden también en este corte cualitativista del AC: [EI Análisis de Contenido] es el conjunto de procedimientos interpretativos de productos comunicativos (mensajes, textos o discursos) que proceden de procesos singulares de comunicación previamente registrados, y que, basados en técnicas a medida, a veces cuantitativas (estadísticas basadas en el recuento de unidades), a veces cualitativas (06) (lógicas basadas en la combinación de categorías) tienen por objeto elaborar y procesar datos relevantes sobre las condiciones mismas en que se han producido aquellos textos, o sobre las condiciones que puedan darse para su empleo posterior (Piñuel, 2002, p.2).

(07) Las preguntas plantean un nivel de profundidad muy diferente en cuanto al análisis de la recepción y sólo buscan ejemplificar diferentes grados y enfoques de estudio.

(08) Hay que recordar el carácter instrumental además de las VVCMM que pueden observarse no sólo a partir de la experiencia directa de la violencia sino también desde un padecimiento indirecto y de un efecto de intimidación constante (Osborne, R., 2009).

(09) Zurbano Berenguer, Belén (2015). Discurso periodístico y violencias contra las mujeres. Tesis doctoral. Universidad de Sevilla. Directores: Dr. Ángel Acosta Romero y Dra. Mar García Gordillo. Inédita.

\section{BREVE SEMBLANZA DE LAS AUTORAS}

Dra. Belén Zurbano Berenguer. Investigadora y profesora en estudios de comunicación, género y violencias. Doctora con mención internacional por la Universidad de Sevilla y miembro del grupo de investigación Estudios de Medios para un Periodismo de Calidad. Responsable del grupo de cooperación AFRICODE (África Cooperación para el Desarrollo) y formadora de la Escuela de Seguridad Pública de Andalucía. Es miembro de la Asociación Española de Investigación de la Comunicación y de la Red Española de Estudios del Desarrollo entre otras sociedades y redes científico-profesionales.

Dra. María del Mar García Gordillo. Profesora contratada doctora. Docente del área de periodismo especializado el el departamento de periodismo II en la Universidad de Sevilla donde imparte docencia en grado, másteres y el doctorado interuniversitario en comunicación. Directora de Comunicación en la Universidad de Sevilla. Responsable del grupo de investigación Estudios de Medios para un Periodismo de Calidad (SEJ- 
001). Miembro de la Asociación Española de Investigación de la Comunicación y de la Sociedad Española de Periodística, además de otras redes científicas.

Ámbitos. Revista Internacional de Comunicación, n.34, edición de otoño, 2016.

Recibido: 27/06/2016

Aprobado: 31/08/2016 